\title{
First report of cucumber mosaic virus isolated from Zinnia elegans in Korea
}

\author{
Dong-Joo Min ${ }^{1} \cdot$ Ji-Soo Park ${ }^{1} \cdot$ Jin-Sung Hong ${ }^{1}$ \\ Received: 27 August 2019 / Accepted: 27 February 2020 / Published online: 6 March 2020 \\ (C) Società Italiana di Patologia Vegetale (S.I.Pa.V.) 2020
}

Keywords Cucumber mosaic virus · Zinnia elegans · Subgroup I · RT-PCR

Zinnia elegans (Asteraceae), is used as an ornamental plant because of the various flower colors and shapes. We collected Z. elegans showing mosaic symptoms from roadside flowerbeds in Chuncheon, Korea. Many viruses including tomato spotted wilt virus (Bakardjieva et al. 1998) have been reported to infect $Z$. elegans. Seven genus-specific primer sets for ilarviruses, potyviruses, fabaviruses, tospoviruses, potexviruses, tobamoviruses and cucumoviruses were used in RT-PCR to detect plant viruses. PCR products were amplified only with the cucumovirus-specific primers (Choi et al. 1999) and cucumber mosaic virus (CMV) was identified by sequencing of these amplicons. Our results confirm that Z. elegans is naturally infected with CMV, as previously reported in other countries (e.g. Shahmohammadi et al. 2015). We named the CMV isolated from Z. elegans as CMV-Ze (GenBank accession Nos. LC390004, LC390005, LC390006). Analysis of $2 b$ and CP sequences showed that CMV-Ze shared $99.1 \%$ and $99.08 \%$ amino acid sequence identity with 2b and CP of CMV-RP15 (KC527699) and CMV-China99/90 (AY611027). Phylogenetic analysis of CPs supports that CMV-Ze is a member of CMV subgroup I. Mechanical inoculation to herbaceous host plants induced mosaic and malformation in upper leaves of

Electronic supplementary material The online version of this article (https://doi.org/10.1007/s42161-020-00532-3) contains supplementary material, which is available to authorized users.

Jin-Sung Hong

jinsunghong@kangwon.ac.kr

1 Department of Bio-resources Sciences, College of Agriculture and Life Sciences, Kangwon National University, Chuncheon 24341, South Korea
$N$. benthamiana, N. tabacum cv. Xanthi nc, N. rustica and $N$. glutinosa by 14 days post inoculation (dpi); necrotic local lesion in Chenopodium quinoa and C. amaranticolor; systemic veinal chlorosis in Cucurbita pepo; and chlorotic spots on upper leaves of Cucumis sativus by 14 dpi. CMV-Ze was confirmed to reinfect $Z$. elegans resulting in mosaic symptoms on upper leaves by $15 \mathrm{dpi}$. These results demonstrate that CMV-Ze has broad host range similar to that reported for other CMV isolates from ornamental plants (Samuitiene and Navalinskiene 2008). To our knowledge, this is the first report of CMV isolated from Z. elegans in Korea.

Funding information This work was supported by the National Research Foundation of Korea (NRF) grant funded by the Korea government (MSIT) (No. 2017R1D1A1B03036553).

\section{References}

Bakardjieva N, Denkova S, Hristova D (1998) Tomato spotted wilt virus on ornamental species in Bulgaria. Biotechnol Biotec Eq 12(2):4952

Choi SK, Choi JK, Park WM, Ryu KH (1999) RT-PCR detection and identification of three species of cucumoviruses with a genusspecific single pair of primers. J Virol Methods 83:67-73

Shahmohammadi N, Dizadji A, Habibi MK, Nateqi M (2015) First report of cucumber mosaic virus infecting Bougainvillea spectabilis, Coleus blumei, Kalanchoe blossfeldiana and Zinnia elegans in Iran. J Plant Pathol 97:394

Samuitienė M, Navalinskienė M (2008) Occurrence of cucumber mosaic cucumovirus on ornamental plants in Lithuania. Žemdirbyste $=$ Agriculture 95(3):135-143

Publisher's note Springer Nature remains neutral with regard to jurisdictional claims in published maps and institutional affiliations. 